\title{
OPTi's Algorithm for Discreteness Determination
}

\author{
Masaaki Wada
}

\section{CONTENTS}

\section{Introduction}

2. Basics of Once-Punctured Torus Groups

3. Indiscreteness Determination by Jørgensen's Inequality

4. The Ford Region for Once-Punctured Torus Groups

5. Discreteness Determination

References
2000 AMS Subject Classification: Primary 20-04;

Secondary 22E40, 30F 40

Keywords: OPTi, algorithm, discrete
We summarize how OPTi draws the parameter space. Each point in the picture of a parameter space corresponds to a group, and the program colors the point according to whether the group is discrete or indiscrete. Applying Jørgensen's inequality to certain sets of generators, OPTi first tries to decide indiscreteness of the group. If the process fails for generators up to a certain depth, the program then tries to construct the Ford region. When it succeeds in constructing the Ford region, Poincaré's polyhedron theorem guarantees the discreteness of the group.

\section{INTRODUCTION}

OPTi [Wada web site] is an application program for the Macintosh that allows one to visualize how isometric circles, the Ford region, the limit set, etc., change as one deforms the once-punctured torus group by moving the mouse.

This article summarizes how OPTi draws the parameter space. In the picture of a parameter space, the points corresponding to discrete groups (quasi-Fuchsian groups) are tiled using various colors, and those corresponding to indiscrete groups are painted out in black. Applying Jørgensen's inequality to certain sets of generators, the program first tries to determine indiscreteness of the groups, and paints the corresponding points black. If a given group satisfies Jørgensen's inequality for generators up to a certain depth, the group is considered to have a high probability of being discrete. The program then tries to construct the Ford region. When the program succeeds in constructing the Ford region, a fundamental region is obtained, and Poincaré's polyhedron theorem guarantees the discreteness of the group. In that case, the corresponding point is painted with various colors according to the combinatorial pattern of the Ford region.

If it is undetermined whether the group corresponding to a point in the parameter space is discrete or indiscrete, the point remains gray. There are two kinds of such gray regions. One is a thin gray region sticking out of a cusp. 
The centerline of such a region contains infinitely many points corresponding to the fundamental groups of cone manifolds. Most of these groups are indiscrete, but the Koebe groups, which are discrete, also reside in this region. The discreteness of groups in this region is difficult to determine using Jørgensen's inequality. In the program, you can choose "Fine" in the ParamSpace menu to apply Jørgensen's inequality for generators of depth up to 1000, and most such gray regions disappear.

Another kind of gray region appears in the middle of an area that is considered obviously discrete. The groups in this region require an appropriate change of generators before the Ford region is constructed. The algorithm in this case is not yet complete.

\section{BASICS OF ONCE-PUNCTURED TORUS GROUPS}

In this section we collect some facts about oncepunctured torus groups that we will need later. For the details, the reader is referred to [Akiyoshi et al. 98, Akiyoshi et al. 00, Akiyoshi et al. 03].

Let $A, B$ denote the meridian and the longitude of the torus. The fundamental group of the once-punctured torus is then the free group generated by $A, B$, but we impose the condition that the puncture be a cusp; namely, the commutator $[A, B]$ is parabolic. Let

$$
x=\operatorname{tr} A, \quad y=\operatorname{tr} B, \quad z=\operatorname{tr} A B,
$$

and assume that the Markov identity

$$
x^{2}+y^{2}+z^{2}=x y z
$$

holds. Dividing both sides of the above by $x y z$, we obtain

$$
a_{0}+a_{1}+a_{2}=1 .
$$

The terms of the left-hand side of this equation, namely,

$$
a_{0}=\frac{x}{y z}, \quad a_{1}=\frac{y}{z x}, \quad a_{2}=\frac{z}{x y},
$$

are called a complex probability.

Conversely, given a complex probability $\left(a_{0}, a_{1}, a_{2}\right)$, we can restore the original group $\langle A, B\rangle$ as in Figure 1 (up to conjugacy of groups). Here, $P$ is the Möbius transformation that represents a 180-degree rotation about the geodesic connecting $\pm i / x$ in the "hyperbolic" upper half-space. The circle centered at the origin is the isometric circle of $P$. We define $Q$ and $R$ similarly. Then $K=R Q P$ is a +1 translation, and we have $A=R Q=K P, B=P Q=K^{-1} R,[A, B]=K^{2}$.

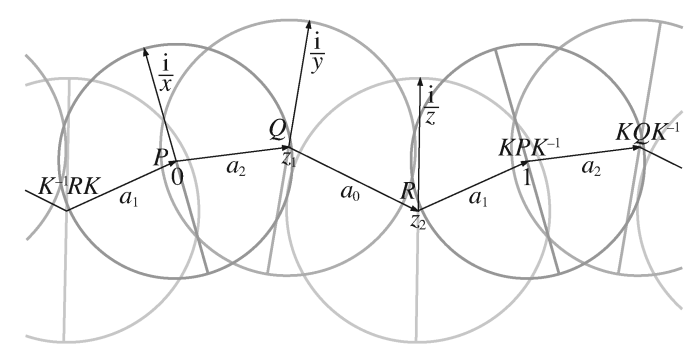

FIGURE 1. Restoring the group from a complex probability.

In the above, the triple $(P, Q, R)$ of elements of order 2 is called an elliptic generator triple. It is a set of generators of the fundamental group of the $(2,2,2, \infty)$-orbifold, which has the once-punctured torus as a branched covering of index 2. Every consecutive triple from the infinite sequence

$$
\begin{array}{r}
\ldots, K^{-1} P K, K^{-1} Q K, K^{-1} R K, P, Q, R, \\
K P K^{-1}, K Q K^{-1}, K R K^{-1}, \ldots
\end{array}
$$

is also an elliptic generator triple.

If $(P, Q, R)$ is an elliptic generator triple, $(P, R, R Q R)$ is also an elliptic generator triple. This transformation corresponds to a change of marking. We call it simply a change of generators (Figure 2). In terms of the trace, this operation corresponds to

$$
(x, y, z) \longmapsto\left(x, z, y^{\prime}\right), \quad\left(y+y^{\prime}=x z\right),
$$

and in terms of complex probability, to

$$
\left(a_{0}, a_{1}, a_{2}\right) \longmapsto\left(a_{0}+a_{2}, \frac{a_{0} a_{1}}{a_{0}+a_{2}}, \frac{a_{1} a_{2}}{a_{0}+a_{2}}\right) .
$$

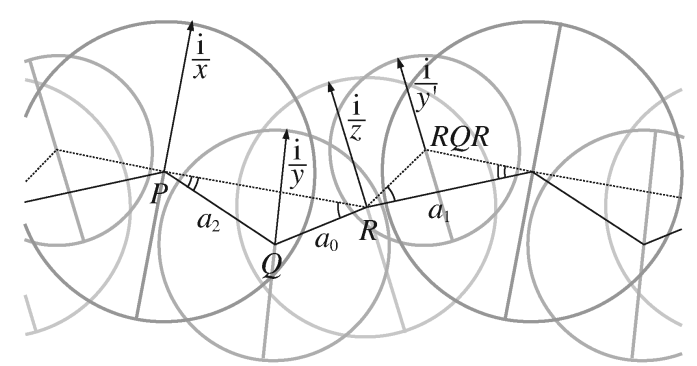

FIGURE 2. Change of generators.

For the infinite sequence obtained from $(P, Q, R)$, there are essentially three different changes of generators, according to which of $P, Q, R$ is omitted. It is known that all the elliptic generators can be obtained by the above operations. Therefore, if we identify elliptic generators belonging to the same infinite sequence and regard them as a point, and regard a change of generators as an edge, we obtain an infinite trivalent tree. 


\section{INDISCRETENESS DETERMINATION BY JØRGENSEN'S INEQUALITY}

Jørgensen's inequality [Jørgensen 76] asserts that if two elements $F, G$ in $\mathrm{SL}(2, \mathbb{C})$ generate a nonelementary discrete group, then

$$
\left|\operatorname{tr}^{2} F-4\right|+|\operatorname{tr}[F, G]-2| \geq 1
$$

We apply this for $F=K, G=P, Q$, or $R$. In this case, the condition of being nonelementary always holds, and we obtain the following criterion: If the radius of the isometric circle of any one of $P, Q, R$ is greater than 1 , then $P, Q, R$ generate an indiscrete group.

The algorithm is the following: Starting from the generators $P, Q, R$, perform changes of generators successively, and apply this criterion to each newly obtained generator. Checking the criterion for generators of depth up to $n$ may seem to require $3 \cdot 2^{n}$ checks, but this is not the case.

Computer experiments reveal that among the three changes of generators applicable to $P, Q, R$, at least one leads to the situation in which the radius of the isometric circle quickly becomes very small as one keeps changing generators. Thus there is no need to apply Jørgensen's inequality in that direction. This fact is supported not only by computer experiments, but also theoretically by Bowditch [Bowditch 98]. Therefore, we can fix a radius, and once the isometric circle of a generator has radius smaller than that, we can stop searching in that direction. This allows us to check Jørgensen's inequality for generators of depth up to $n$ essentially in linear time.

This check is quite effective. The groups not determined to be indiscrete by this check up to, say, depth 1000 are almost $100 \%$ discrete, except for the special case mentioned below. This is the author's feeling acquired through various experiments using OPTi. In fact, in the ParamSpace menu in OPTi, "Coarse," "Medium," and "Fine" correspond respectively to checks of depth 10, 100, 1000, and while the "Coarse" setting may show some gray regions, the "Medium" setting already leaves very little gray.

Let us now consider the special case in which the group is indiscrete and yet passes the check by Jørgensen's inequality. This corresponds to the thin curved gray region coming out of a cusp in the parameter space shown by OPTi. Choosing points in such a region in OPTi and investigating the groups reveals that for such a group some successive changes of generators lead to a kind of "rotating pattern," and the radius of the isometric circles becomes neither large nor small. For most such groups, sufficiently many changes of generators produce an isometric circle of radius greater than 1 after all, and the group is determined to be indiscrete. That is, the larger we make the depth $n$ of the check by Jørgensen's inequality, the thinner such a gray region becomes.

However, in the center of such a region, there supposedly lies a 1-dimensional family of groups for which some successive changes of generators produce a rotating pattern ad infinitum, and thus such a region never disappears completely. There are very special cases of these groups in which $k$ changes of generators put the generators back into their original positions. These are the Koebe groups. The Koebe groups are discrete, and satisfy Jørgensen's inequality. Between the Koebe groups, it is supposed that there lies a 1-dimensional family of groups for which changes of generators produce, so to speak, an irrational rotation pattern. But nothing is known about this theoretically.

\section{THE FORD REGION FOR ONCE-PUNCTURED TORUS GROUPS}

If a group has not been determined to be indiscrete by passing the above check by Jørgensen's inequality, the program tries to construct the Ford region, which will be explained in detail in the next section. But first, let us give an account of Jørgensen's theory of the Ford region for once-punctured torus groups.

One way of defining the Ford region is to consider all the isometric hemispheres of the elements of the group not fixing infinity, and to define these as the boundary pattern (projected down onto the complex plane) of the union of these solid hemispheres. In the case of oncepunctured torus groups, it is known that we may restrict to the isometric hemispheres of elliptic generators only.

Figure 3 shows an example of a typical once-punctured torus group. Solid lines represent the Ford region, and various dotted lines represent complex probabilities. Starting from the lowermost complex probability in the picture, one can see how generators are changed four times to reach the topmost complex probability. Looking at it carefully, one notices that the triangle pattern produced by the complex probabilities is dual to the Ford region in the combinatorial sense.

Jørgensen [Jørgensen 03] claims that the Ford region of every geometrically finite once-punctured torus group is, as in this example, combinatorially dual to the triangle pattern obtained by applying an appropriate finite changes of generators to some complex probability. We leave the details to [Akiyoshi et al. 03]. Therefore, of 


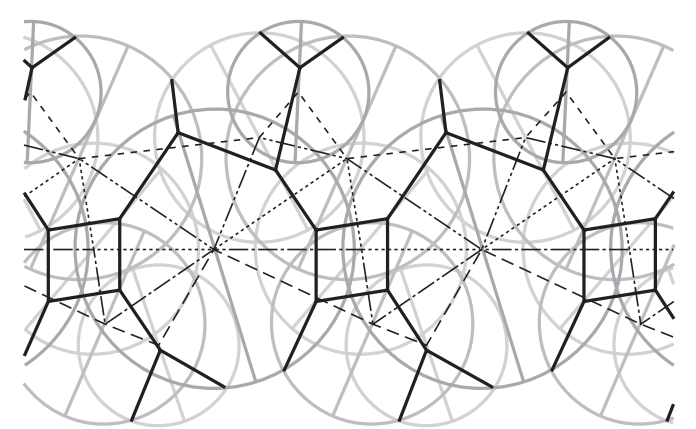

FIGURE 3. A typical once-punctured torus group.

the isometric hemispheres of infinitely many sequences of elliptic generators, only the isometric hemispheres of those along a finite path in the infinite tree are "visible from above." Let us call these sequences of elliptic generators (and the complex probabilities) corresponding to the Ford region the Ford sequence.

Now, according to Jørgensen's theory, given a complex probability belonging to the Ford sequence, one can determine as follows which of the three possible changes of generators lead to the upper and lower adjacent complex probabilities.

First, draw all the isometric circles of the elliptic generators corresponding to the given complex probability. If all these circles face the upper region in the complex plane along arcs, the given complex probability corresponds to the upper end of the Ford sequence (Figure 4). For each edge of the "line graph" corresponding to the complex probability, consider the triangle defined by the two endpoints and the upper intersection of the isometric circles of the two generators corresponding to these endpoints. Then, the above condition is equivalent to the condition that these triangles do not intersect in the interior.

When we draw the triangles along the line graph, if a pair of adjacent triangles intersect in the interior,

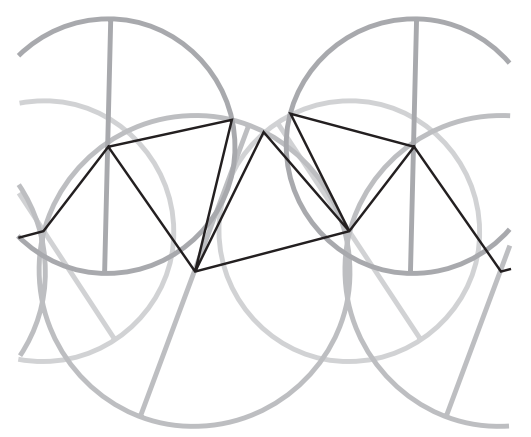

FIGURE 4. Upper end of the Ford sequence. The triangles do not intersect.

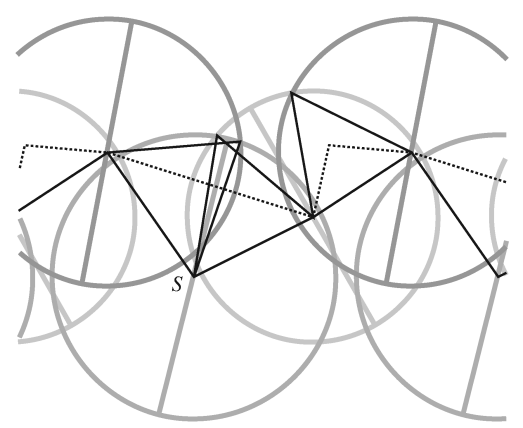

FIGURE 5. Case in which two triangles intersect. The change of generators discarding the generator "S" between the two intersecting triangles leads to the upper adjacent complex probability.

the change of generators that throws away the generator corresponding to the common endpoints of the adjacent edges leads to the upper adjacent complex probability in the Ford sequence (Figure 5).

If two adjacent pairs of triangles both intersect, we need to decide which of the generators to throw away. This can be done as follows. Let $a, b, c$ be consecutive edges of the line graph corresponding to the given complex probability, and assume that both the triangles for $a, b$ and those for $b, c$ intersect in the interior (Figure 6). Draw the line segment $\alpha$ connecting the two intersection points of the isometric circles of the generators corresponding to the two endpoints of the edge $a$. Also draw analogous line segments $\beta, \gamma$ for the edges $b, c$. Then, the line segments $\alpha$ and $\gamma$ both intersect the line segment $\beta$ in the upper side of the edge $b$. Now, according to which of $\alpha$ and $\gamma$ meets $\beta$ at a closer point to the edge $b$, the change of generators discarding the generator corresponding to the endpoint of $b$ on the side of $a$ or $c$ leads to the upper adjacent complex probability in the Ford sequence.

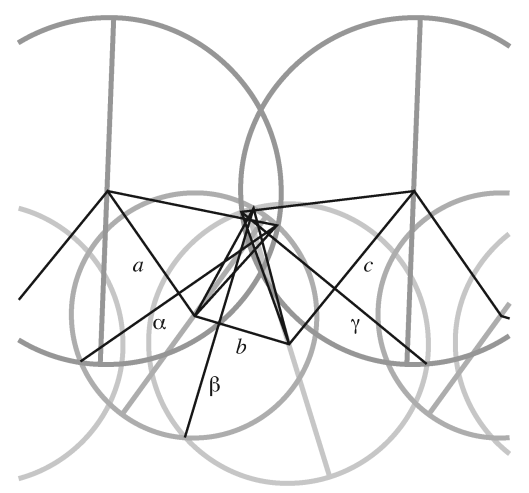

FIGURE 6. Case that two pairs of triangles intersect. 
In the above, we have only described how to determine the upper adjacent complex probability. The lower adjacent complex probability in the Ford sequence can also be determined by a similar criterion. Therefore, if we have a complex probability in the Ford sequence, we can determine the Ford sequence by computing the upper and lower adjacent complex probabilities one by one, and thus construct the Ford region.

Note that this can be done only under the assumption that a complex probability in the Ford sequence is given. In general, when an arbitrary complex probability is given, no easy way of determining whether it belongs to the Ford sequence is known.

\section{DISCRETENESS DETERMINATION}

OPTi tries to determine discreteness as follows. Given a complex probability, assume that it belongs to the Ford sequence, and consider computing the upper and lower adjacent complex probabilities as explained in the previous section. We first need to draw the triangles above and below the line graph obtained from the complex probability. There are cases in which the triangle inequality fails to hold, and we cannot construct the triangles. In such a case, the isometric hemisphere of the generator corresponding to the common endpoints of two adjacent short edges is completely covered by the isometric hemispheres of the adjacent generators. Hence the generator is obviously not a member of the Ford sequence. Therefore we perform the change of generators that discards such a generator.

If the triangle inequality holds everywhere, we construct the triangles above and below and try to compute the upper and lower adjacent complex probabilities. There are cases in which first computing the upper adjacent complex probability and then its lower adjacent one does not give the original complex probability. If this happens, we know that the original complex probability was not a member of the Ford sequence. In such a case, we throw away all the complex probabilities thus far obtained and restart the algorithm from the point where the inconsistency occurred.

This way we keep constructing a sequence of complex probabilities while paying attention to the consistency mentioned above. If we have obtained a consistent sequence including the upper and lower ends satisfying the criteria for the Ford sequence explained in the previous section, we can show that the complement in the hyperbolic space of the union of the (solid) isometric hemispheres of the generators appearing in the sequence satisfies the conditions of Poincaré's polyhedron theorem, and hence is a fundamental region. Therefore the group is discrete.

Actually, a problematic situation may occur in the above process. Namely, the line graph corresponding to the complex probability appearing in the process may have self-intersections. It is not true that such a complex probability always comes from an indiscrete group. In fact, if we start from a complex probability belonging to the Ford sequence of a discrete group, and perform changes of generators several times in the third direction, which does not correspond to the Ford sequence, we often obtain a complex probability of self-intersecting type. Now if we are given such a complex probability of self-intersecting type at the beginning, we know that the group itself is discrete.

It is obvious that a complex probability of selfintersecting type is never a member of the Ford sequence. The question is how we can determine which of the three possible changes of generators leads to the Ford sequence. While Bowditch [Bowditch 98] gives an answer to the question theoretically, it is an open question at the moment whether a practical method can be implemented. To make the point clear, when a complex probability of self-intersecting type appears in the process, OPTi draws the corresponding point gray. The gray regions appearing here and there in the picture of parameter space are such regions.

\section{REFERENCES}

[Akiyoshi et al. 98] H. Akiyoshi, M. Sakuma, M. Wada, and Y. Yamashita. "Punctured Torus Groups and TwoParabolic Groups." RIMS Kokyuroku 1065, Analysis and Geometry of Hyperbolic Spaces (1998), 61-73.

[Akiyoshi et al. 00] H. Akiyoshi, M. Sakuma, M. Wada, and Y. Yamashita, "Ford Domains of Punctured Torus Groups and Two-Bridge Knot Groups." RIMS Kokyuroku 1163, Hyperbolic Spaces and Related Topics II (2000), 67-77.

[Akiyoshi et al. 03] H. Akiyoshi, M. Sakuma, M. Wada, and Y. Yamashita, "Jørgensen's Picture of Punctured Torus Groups and Its Refinement." In Kleinian Groups and Hyperbolic 3-Manifolds, pp. 247-273. London: Lond. Math. Soc. LNS 299 (2003).

[Bowditch 98] B. H. Bowditch, "Markoff Triples and Quasifuchsian Groups." Proc. London Math. Soc. 77 (1998), 697736.

[Jørgensen 76] T. Jørgensen, "On Discrete Groups of Möbius Transformations." Amer. J. Math. 98 (1976), 739-749. 
[Jørgensen 03] T. Jørgensen, "On Pairs of Once-Punctured Tori." Kleinian Groups and Hyperbolic 3-Manifolds, pp. 183-207. London: Lond. Math. Soc. LNS 299 (2003).
[Wada web site] M. Wada, OPTi. Available online at http://vivaldi.ics.nara-wu.ac.jp/ ${ }^{\sim}$ wada/OPTi/.

Masaaki Wada, Department of Information and Computer Sciences, Nara Women's University, Nara 630-8506, Japan (wada@ics.nara-wu.ac.jp)

Received November 15, 2004; accepted in revised form October 27, 2005. 